Pacific Journal of Mathematics

WILD ARCS IN THREE-SPACE. II. AN INVARIANT OF 


\title{
WILD ARCS IN THREE-SPACE 2: AN INVARIANT OF NON-ORIENTED LOCAL TYPE
}

\author{
JAMES M. MCPHERSON
}

\begin{abstract}
An invariant of the oriented local type of a Fox-Artin arc has already been developed in the previous paper in this sequence. This paper is concerned with finding an invariant of the non-oriented local type of an arc whose only wild point is an endpoint, where the arc has penetration index at least five, and where the penetration index with respect to solid tori is equal to one.
\end{abstract}

This paper contains most of the results announced in $\S 3$ of [5], and forms the first chapter of the author's Ph. D. thesis [4], written under the supervision of Professor N. Smythe.

1. Preliminaries. Rather than work with 3-cells as in [6], our cutting and pasting arguments will be applied to sequences of solid tori, making use of the theorems of H. Schubert $([7], \S \S 16,17)$. The notations $\mathrm{Bd}, \mathrm{Cl}$, Int, $N(X), \nu(k \cap X)$ are used as in [6].

1. $k$ is an oriented arc in Euclidean 3-space $R^{3}$ (the orientation of $R^{3}$ is fixed), and $k$ is tame except at the endpoint $p$.

2. A handlebody of genus $g$ (Henkelkörper von Geschlechte $g$, [8], p. 219) is a tame closed regular neighbourhood of a wedge of $g$ circles. If $g=1$, such a handlebody is a solid torus (i.e., the topological product of $S^{1}$ with a 2-disc, called "Vollring" in [7]), and if $g=0$ we have a 3 -cell.

The penetration index $P_{g}(k, p)$ of $k$ at $p$, relative to handlebodies of genus $g$, is the smallest integer $n$ such that there exist arbitrarily small neighbourhoods of $p$ which are handlebodies of genus $g$, each meeting $k$ on its boundary in $n$ points. When there is no danger of confusion, we write $P_{g}(k, p)=P_{g}$. (Note: It is not known whether $P_{g}(k, p)$ is the same as the penetration index of $k$ relative to surfaces of genus $g$, as defined by B. J. Ball in [1]-cf. question 1 of [5].)

$P_{1}$ is called the toral penetration index; $P_{0}$ is called the 3-cell penetration index and is the "nice penetration index" defined by Henderson ([3], p. 470).

As in [6], we choose a 3-cell neighbourhood $E_{0}$ of $p$, chosen so that if $E \subset$ Int $E_{0}$ is any other such neighbourhood, then Bd $E$ meets $k$ in at least as many points as does Bd $E_{0}$.

3. For the notion of cofinality of two sequences, see [6], §2.

Two arcs $k_{1}$ and $k_{2}$ have the same non-oriented local type at points $p_{1}$ and $p_{2}$ if there exist neighbourhoods $U_{i}$ of $p_{i}$, and a homeomorphism 
$h$ of $U_{1}$ to $U_{2}$ which takes $\left(U_{1} \cap k_{1}, p_{1}\right)$ to $\left(U_{2} \cap k_{2}, p_{2}\right)$.

In these terms, then, the aim of this paper is to find an invariant of the non-oriented local embedding type of $k$ in $E_{0}$, if $P_{1}(k, p)=1$ and $P_{0}(k, p) \geqq 5$. In some cases, we may associate an infinite sequence of solid tori with $k$, and show that the cofinality class of the knot types (q.v., no. 4) of these solid tori is an invariant of the non-oriented local type of $k$ at $p$. Analogous results have been obtained in [4] for arcs or simple closed curves with $P_{1}=2$ and $P_{0} \geqq 8$ : for convenience, however, we shall restrict ourselves to arcs which are wild at an endpoint.

4. Let $V$ be a solid torus. A meridian of $\mathrm{Bd} V$ is a curve which bounds a disc in $V$ but not on Bd $V$; such a disc will be called a meridian disc of $V$. A longitude of $\mathrm{Bd} V$ is any curve on $\mathrm{Bd} V$ which is nullhomologous in $\mathrm{Cl}\left(R^{3}-V\right)$ but not on $\mathrm{Bd} V$; a core of $V$ is a curve homologous in $V$ to a longitude of $\mathrm{Bd} V$. If $\lambda$ is a longitude of $V$, we denote by $\kappa(V)$ the non-oriented knot type of $\lambda$, and say that $V$ is knotted with knot type $\kappa(V)$.

Let $U$ and $V$ be solid tori with $U \subset V$. The order of $U$ in $V$, denoted $O(U, V)$, is the minimum number of intersections of a meridian disc of $V$ with a longitude of $U$ (this integer depends on neither the longitude nor the meridian disc chosen). If $\mathrm{T} \subset U$ is another solid torus, then

$$
O(T, V)=O(T, U) \cdot O(U, V)
$$

([7], p. $172 \mathrm{ff})$.

We write $U \prec V$ iff $O(U, V)=0$, that is, if and only if $U$ lies in a 3-cell in the interior of $V$.

The knot $\kappa_{1}$ is a companion of the knot $\kappa_{2}$ if a solid torus $V_{2}$, such that $\kappa\left(V_{2}\right)=\kappa_{2}$, may be embedded with nonzero order in the interior of a solid torus $V_{1}$ with $\kappa\left(V_{1}\right)=\kappa_{1}$. $\kappa_{1}$ is a factor of $\kappa_{2}$ if $V_{2}$ may be embedded in $V_{1}$ with order one.

Two solid tori $V_{1}, V_{2}$, with $V_{2} \subset \operatorname{Int} V_{1}$, are concentric if the closure of $V_{1}-V_{2}$ is homeomorphic to $I \times \mathrm{Bd} V_{1}$, where $I$ is the unit interval. Theorem 3 of [2] asserts that $V_{1}$ and $V_{2}$ are concentric iff $\kappa\left(V_{1}\right)=$ $\kappa\left(V_{2}\right)$ and $V_{2}$ has order one in $V_{1}$.

5. A $k$-torus is a non-oriented tame closed solid torus $V \subset \operatorname{Int} E_{0}$, which contains $p$ in its interior, and meets $k$ on its boundary in one point only, at which it is pierced by $k$. $k$-tori

A containing sequence (in $E_{0}$ ) for a $k$-torus $V$ is a sequence of

$$
V=V_{n} \prec V_{n-1} \prec \cdots \prec V_{0} \subset E_{0}
$$

with the property: if $U$ is any $k$-torus, $V_{i} \subset U \subset V_{i-1}$, then either $V_{i}$ 
has nonzero order in $U$, or $U$ has nonzero order in $V_{i-1}$. If $V_{0} \subset U \subset$ $E_{0}$, we require that $V_{0}$ have nonzero order in $U$. A containing sequence is of length $n$ if $V=V_{n}$.

A constructing sequence for $k$ (in $E_{0}$ ) is a sequence of $k$-tori $E_{0} \supset$ $V_{0} \succ V_{1} \succ V_{2} \succ \cdots$ such that $\cap V_{i}=p$, and for each $i$,

$$
V_{i} \prec V_{i-1} \prec \cdots \prec V_{0} \subset E_{0}
$$

is a containing sequence for $V_{i}$.

A $k$-sequence is a constructing sequence with the further property that if $V$ is a $k$-torus with $V_{i} \subset V \subset V_{i-1}$, and $V$ has nonzero order in $V_{i-1}$, then $V$ and $V_{i-1}$ are concentric. $V$ and $V_{i-1}$ are equally knotted, therefore: thus a $k$-sequence is a constructing sequence in which the $k$-tori occurring are as "tightly knotted" (stärker verknotet, [7], p. 211) as possible.

An example may help to highlight the difference between a constructing sequence and a $k$-sequence. The arc $k$ of Figure 1(a) may

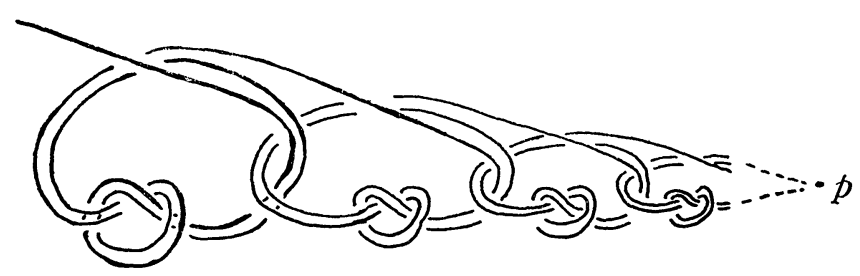

(a)

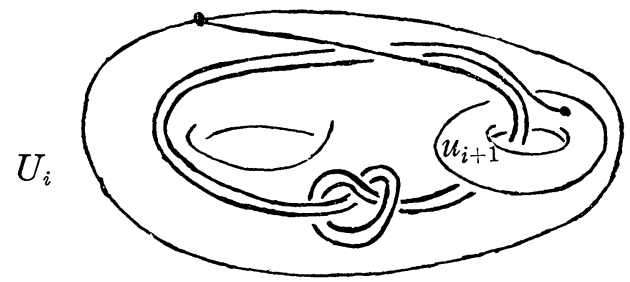

(b)

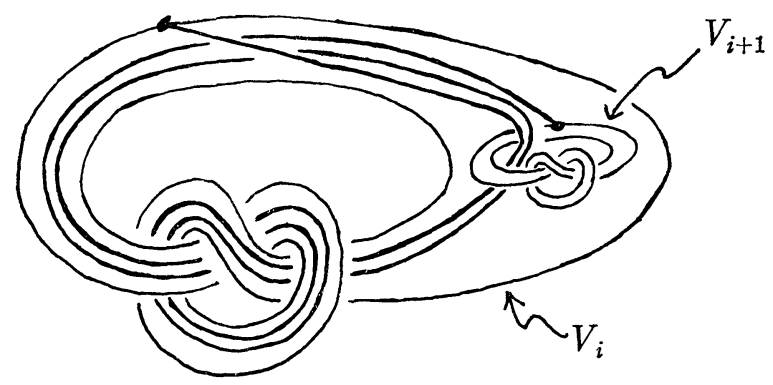

(c)

FiguRE 1 
be constructed from solid tori placed in relation to each other and to $k$ as shown in Figure $1(\mathrm{~b})$. But one can replace $U_{i}$ and $U_{i+1}$ by solid tori $V_{i}$ and $V_{i+1}$, as shown in Figure 1(c), to construct the same arc. A sequence of solid tori of the type of $U_{i}, U_{i+1}$ will suffice to construct the arc, but will not be a $k$-sequence.

6. Two $k$-tori $U$ and $V$ are $k$-similar, ${ }^{1}$ if there exists a pair of $k$-tori $A_{1}$ and $A_{2}$, such that $A_{2} \subset \operatorname{Int}(U \cap V)$, Int $A_{1} \supset U \cup V$, and $A_{2}$ has nonzero order in $A_{1}$. We say that $U$ is $k$-similar to $V$ via the $k$-tori $A_{1}$ and $A_{2}$.

We note that if $U$ lies in the interior of $V$ with nonzero order, then $U$ and $V$ are $k$-similar. Similarly, if $U$ and $V$ are $k$-similar via the $k$-tori $A_{1}$ and $A_{2}$, then any two of $U, V, A_{1}, A_{2}$ are $k$-similar.

$k$-similarity is a reflexive and symmetric relation, but unfortunately is not transitive. In Figure 2, $T$ and $U$ are $k$-similar, $U$ and $V$ are $k$-similar, but $T$ and $V$ cannot be $k$-similar.

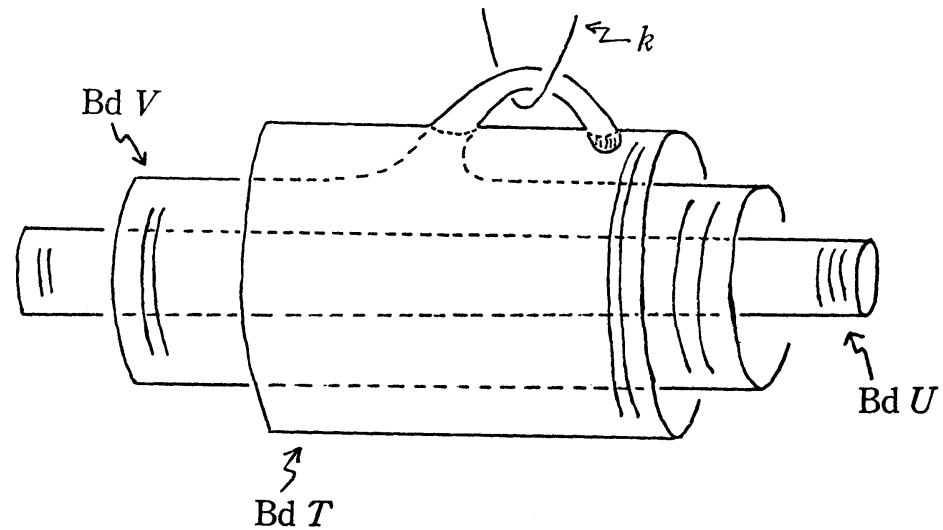

FIGURE 2

QUESTION (Fox). Is it possible to replace $k$-similarity by a transitive relation?

7. We come to two simple but important lemmas.

LEMma 1. If $V$ is a k-torus, every meridian disc of $V$ meets $k$ in at least two points (we assume that our meridian discs do not contain p). If $V$ is unknotted, every disc bounded by a longitude of $\mathrm{Bd} V$ meets $k$ in at least two points.

Proof. Let $D$ be a meridian disc of $V$. Then $V$-\{an open regular neighbourhood of $D$ \} is a 3-cell neighbourhood of $p$ which meets $k$ on

1 The use of the notation $\succ$ and the terms "containing sequence" and " $k$-similar" both here and in [6] should not lead to any confusion. If the two uses of any of these terms should occur together in one paper, context will decide which use is intended. This paper will not use the terms as they are used in [6]. 
its boundary in $P_{1}+2 N(k \cap D)$ points. Hence

$$
P_{1}+2 N(k \cap D)=1+2 N(k \cap D) \geqq P_{0} \geqq 5,
$$

and the first result follows. The proof of the second result is similar.

LemmA 2. Let $U, B_{1}, B_{2}$ be k-tori with $B_{2} \subset U \subset B_{1}$, and let $U^{*}$ be $a k$-torus which is $k$-similar to $U$ via $k$-tori $A_{1}$ and $A_{2}$, with $B_{2} \subset A_{i} \subset B_{1}$. Then $B_{2}$ has nonzero order in $U^{*}$ iff it has nonzero order in $U$, and $U^{*}$ has nonzero order in $B_{1}$ iff $U$ has nonzero order in $B_{1}$.

Proof. The numbers $O\left(U^{*}, A_{1}\right), O\left(U, A_{1}\right), O\left(A_{2}, U^{*}\right)$, and $O\left(A_{2}, U\right)$ are all nonzero. The result follows from the relations (cf. no. 4):

$$
O\left(B_{2}, U^{*}\right) \cdot O\left(U^{*}, A_{1}\right)=O\left(B_{2}, A_{1}\right)=O\left(B_{2}, U\right) \cdot O\left(U, A_{1}\right)
$$

and

$$
O\left(A_{2}, U^{*}\right) \cdot O\left(U^{*}, B_{1}\right)=O\left(A_{2}, B_{1}\right)=O\left(A_{2}, U\right) \cdot O\left(U, B_{1}\right)
$$

2. Some surgical lemmas. The following lemmas will be useful in our cutting and pasting arguments. In each, $U$ and $V$ are $k$-tori whose boundaries are in general position. We are also assuming that none of the curves of $\mathrm{Bd} U \cap \mathrm{Bd} V$ contains any points of $k$-this may be achieved by a small ambient isotopy of $E_{0}$, if necessary.

Lemma 3. Let $D$ and $D^{\prime}$ be discs on the boundaries of $U$ and $V$ respectively, such that $\mathrm{Bd} D=\mathrm{Bd} D^{\prime}$, and suppose further that the interior of $D$ does not meet the boundary of $V . \quad D \cup D^{\prime}$ is the boundary of a 3-cell $S ; S$ does not contain $p$, and the solid torus $V^{\prime}$ is a $k$-torus, where

$$
\begin{aligned}
& V^{\prime}=V \cup S \text { if } D \subset \mathrm{Cl}\left(E_{0}-V\right) \\
& V^{\prime}=\mathrm{Cl}(V-S) \text { if } D \subset V .
\end{aligned}
$$

Moreover, $V$ and $V^{\prime}$ are equally knotted.

Proof. To show that $S$ cannot contain $p$, we show that $N(k \cap$ $\operatorname{Bd} S)<P_{0}$. Now

$$
\begin{aligned}
N(k \cap \mathrm{Bd} S) & =N(k \cap D)+N\left(k \cap D^{\prime}\right) \\
& \leqq N(k \cap \mathrm{Bd} U)+N(k \cap \mathrm{Bd} V)=1+1<5 \leqq P_{0} .
\end{aligned}
$$

So $p \notin S$, and $p$ must therefore lie in $V^{\prime}$. If $V^{\prime}$ is to be a $k$-torus, we must show that $N\left(k \cap \mathrm{Bd} V^{\prime}\right)=P_{1}=1$, and for this it is sufficient to show that $N(k \cap D)=N\left(k \cap D^{\prime}\right)$, because $\mathrm{Bd} V^{\prime}=D \cup\left(\operatorname{Bd} V-D^{\prime}\right)$. $V^{\prime}$ is a small torus containing $p$ in its interior, so $N\left(k \cap \mathrm{Bd} V^{\prime}\right) \geqq$ 
$N(k \cap \operatorname{Bd} V)=P_{1}(k, p)$; that is, $N(k \cap D) \geqq N\left(k \cap D^{\prime}\right)$. We have two cases to consider:

$N(k \cap D)=1$. Since $p \notin S, \nu(k \cap \mathrm{Bd} S)=0$. Now

$$
\nu(k \cap \mathrm{Bd} S)=\nu(k \cap D)+\nu\left(k \cap D^{\prime}\right)=\nu\left(k \cap D^{\prime}\right) \pm 1=0,
$$

so $\nu\left(k \cap D^{\prime}\right)=\mp 1$. $D^{\prime}$ therefore meets $k$ in an odd number of points; since $D^{\prime}$ is on the boundary of a torus which meets $k$ in one point only, $D^{\prime}$ can contain at most one point of $k$. Hence $N\left(k \cap D^{\prime}\right)=1=$ $N(k \cap D)$.

$N(k \cap D)=0$. Then we have

$$
\begin{aligned}
1 \leqq N\left(k \cap \mathrm{Bd} V^{\prime}\right) & =N\left(k \cap\left(\mathrm{Bd} V-D^{\prime}\right)\right)+N(k \cap D) \\
& =N\left(k \cap\left(\operatorname{Bd} V-D^{\prime}\right)\right) \leqq N(k \cap \operatorname{Bd} V)=1 ;
\end{aligned}
$$

thus $N\left(k \cap\left(\mathrm{Bd} V-D^{\prime}\right)\right)=1=N(k \cap \mathrm{Bd} V)$, so $D^{\prime}$ contains no points of $k$. That is, $N\left(k \cap D^{\prime}\right)=0=N(k \cap D)$.

In both cases above, then, $V^{\prime}$ is a $k$-torus. In fact, $V^{\prime}$ and $V$ are equivalently knotted, for there is an ambient isotopy $h_{t}$ of $E_{0}$ which fixes everything outside an open regular neighbourhood of $S$, and takes $V$ to $V^{\prime}$.

Although $\mathrm{Bd} V$ is in general position with respect to $\mathrm{Bd} U, \mathrm{Bd} V^{\prime}$ is not, for the disc $D$ is one component of $\mathrm{Bd} U \cap \mathrm{Bd} V^{\prime}$ We may put the surfaces into general position by a small "push" in the appropriate direction: if $D \subset V$, take $V^{\prime \prime}=V-$ an open regular neighbourhood of $S\}$, and take $V^{\prime \prime}=V \cup\{$ a closed regular neighbourhood of $S\}$ if $D \subset \mathrm{Cl}\left(E_{0}-V\right)$. Then $\mathrm{Bd} V^{\prime \prime}$ is in general position with respect to $\mathrm{Bd} U$, and by requiring our regular neighbourhoods to be sufficiently small, we may ensure that $V^{\prime \prime}$ has the properties claimed for $V^{\prime}$. It is in this sense that we will be applying Lemma 3.

Lemma 4. Let $A$ and $A^{\prime}$ be annuli on the boundaries of $U$ and $V$ respectively, such that $\mathrm{Bd} A=\mathrm{Bd} A^{\prime}$, and suppose further that the interior of $A$ does not meet the boundary of $V$. If $A \cup A^{\prime}$ is the boundary of a solid torus which contains $p$ in its interior, then this torus $V^{\prime}$ is a k-torus.

Proof. We need only show that $k$ meets $V^{\prime}$ on its boundary in one point. Since $N(k \cap \mathrm{Bd} V)$ is minimal, $N\left(k \cap \mathrm{Bd} V^{\prime}\right) \geqq N(k \cap \mathrm{Bd} V)$, that is $N(k \cap A) \geqq N\left(k \cap\left(\mathrm{Bd} V-A^{\prime}\right)\right)$ (for, to obtain $V^{\prime}$, we replaced the annulus $\mathrm{Bd} V-A^{\prime}$ on $\mathrm{Bd} V$ by the annulus $A$ ). Thus we need only show that $N(k \cap A)=N\left(k \cap\left(\mathrm{Bd} V-A^{\prime}\right)\right)$-but this follows as in Lemma 3.

We may put $\mathrm{Bd} V^{\prime}$ into general position with respect to $\mathrm{Bd} U$, by 
taking $V^{\prime \prime}=V^{\prime}-\{$ an open regular neighbourhood of $A\}$ if $A \subset V$, or by taking $V^{\prime \prime}=V^{\prime} \cup$ a closed regular neighbourhood of $\left.A\right\}$ if $A \subset$ $\mathrm{Cl}\left(\mathrm{E}_{0}-V\right)$. By requiring our regular neighbourhoods to be sufficiently small, we may ensure that $V^{\prime \prime}$ is a $k$-torus. It is in this sense that we will be applying Lemma 4 .

LEMma 5. There exists a k-torus $V^{*}$ with the properties:

(i) $V^{*}$ and $V$ are k-similar,

(ii) $\mathrm{B} d V^{*} \cap \mathrm{Bd} U=\varnothing$, and

(iii) $\kappa(V)$ is a companion of $\kappa\left(V^{*}\right)$ (cf. $\S 1$, no. 4).

Moreover, if $B_{1}$ and $B_{2}$ are k-tori with $B_{2} \subset \operatorname{Int}(U \cap V)$, Int $B_{1} \supset$ $U \cup V$, then we may choose $V^{*}$ so that $B_{2} \subset \operatorname{Int} V^{*} \subset V^{*} \subset \operatorname{Int} B_{1}$.

Note. The essence of this lemma is that given two $k$-tori $U$ and $V$, we can leave $U$ fixed and cut around the boundary of $V$ to obtain another $k$-torus $V^{*}$ whose boundary does not meet $\mathrm{Bd} U$; and all this may be done without moving $\mathrm{Bd} V$ too far.

Proof. Let $\mathscr{V}$ be the class of all $k$-tori $T$ which are $k$-similar to $V, B_{2} \subset$ Int $T \subset T \subset \operatorname{Int} B_{1}$, such that $\kappa(V)$ is a companion of $\kappa(T)$ and such that $\mathrm{Bd} T$ is in general position with respect to $\mathrm{Bd} U . V \in \mathscr{V}$, so $\mathscr{V}$ is not empty; therefore there exists a $k$-torus $V^{*}$ in $\mathscr{V}$ whose boundary intersects $\mathrm{Bd} U$ in fewer curves than does the boundary of any other $T \in \mathscr{Y}$. We intend to show that $\mathrm{Bd} V^{*} \cap \mathrm{Bd} U=\varnothing$.

(a) Suppose some intersection curve is null-homologous on $\mathrm{Bd} U$. We may choose one such curve, $\sigma$ say, which bounds a disc $D$ not containing any other intersection curves: Int $D \cap \mathrm{Bd} V^{*}=\varnothing$. Either (i) $D \subset V^{*}$, or (ii) $D \subset \mathrm{Cl}\left(E_{0}-V^{*}\right)$.

(i) $D \subset V^{*}$. $D$ cannot be a meridian disc of $V^{*}$, since such discs meet $k$ in at least two points, by Lemma 1 , and $N(k \cap D) \leqq$ $N(k \cap \mathrm{Bd} U)=1$. So $\sigma$ must be null-homologous on $\mathrm{Bd} V^{*}$.

(ii) $D \subset \mathrm{Cl}\left(E_{0}-V^{*}\right)$. If $V^{*}$ is non-trivially knotted, $\sigma$ must be null-homologous on $\mathrm{Bd} V^{*}$, for a curve which is not nullhomologous on $\mathrm{Bd} V^{*}$ cannot bound a disc in $\mathrm{Cl}\left(E_{0}-V^{*}\right)$. If $V^{*}$ is unknotted, $D$ cannot be a longitude disc, because such discs meet $k$ in at least two points and $D$ meets $k$ in at most one point.

It follows in both cases that $\sigma$ must also be null-homologous on $\mathrm{Bd} V^{*}$, bounding a disc $D^{\prime}$, say. $\mathrm{D} \cup D^{\prime}$ is the boundary of a 3-cell $S$, and we may apply Lemma 3 to obtain a $k$-torus $V^{\prime}$ with $\kappa\left(V^{\prime}\right)=$ $\kappa\left(V^{*}\right)$, and whose boundary is in general position with respect to $\mathrm{Bd} U$; in fact, $\mathrm{Bd} V^{\prime}$ meets $\mathrm{Bd} U$ in fewer intersection curves than does $\mathrm{Bd} V^{*}$, since the curve $\sigma=\mathrm{Bd} D$ in particular has been eliminated. Also, we may choose our regular neighbourhoods of $S$ (see the remark after Lemma 3 ) so that $V^{\prime}$ lies in the interior of $B_{1}$, and contains $B_{2}$ 
in its interior. $V^{\prime}$ will be an element of $\mathscr{V}$ if we can prove that $V^{\prime}$ and $V$ are $k$-similar-the existence of this $k$-torus $V^{\prime} \in \mathscr{V}$ will then contradict the minimality assumption involved in our choice of $V^{*} \epsilon$ $\mathscr{V}$. With this contradiction, we must conclude that no intersection curve is null-homologous on $\mathrm{Bd} U$.

Suppose that $V^{*}$ and $V$ are $k$-similar via the $k$-tori $A_{1}^{*}$ and $A_{2}^{*}$. Then after general positioning, $\mathrm{Bd} V^{\prime} \cap \mathrm{Bd} A_{i}^{*}$ consists of a finite number of simple closed curves $\alpha_{1}, \cdots, \alpha_{s}$, and we note that each of these curves lies in the interior of $D$. Thus each of the curves $\alpha_{j}$ is null-homologous on $\mathrm{Bd} A_{i}^{*}$, by Lemma 1 .

Note that $\operatorname{Bd} V^{\prime}$ does not meet $\operatorname{Bd} A_{2}^{*}$ if $V^{\prime} \supset V^{*} \cup S \supset V^{*}$, and that Bd $V^{\prime}$ does not meet $\mathrm{Bd} A_{1}^{*}$ if $V^{\prime} \subset \mathrm{Cl}\left(V^{*}-S\right) \subset V^{*}$. Thus we need only "adjust" the appropriate $k$-torus $A_{i}^{*}$ to obtain a $k$-torus $A_{i}$ such that $V^{\prime}$ and $V$ are $k$-similar via $A_{1}$ and $A_{2}^{*}$ or via $A_{1}^{*}$ and $A_{2}$, whichever is appropriate.

We may choose a curve, $\alpha_{1}$ say, which bounds a disc $D\left(\alpha_{1}\right)$ on $\operatorname{Bd} V^{\prime}$, which contains no other " $\alpha$-curves". Let $D^{\prime}\left(\alpha_{1}\right)$ be the corresponding disc on the boundary of $A_{i}^{*}$; then $D\left(\alpha_{1}\right) \cup D^{\prime}\left(\alpha_{1}\right)$ is the boundary of a 3-cell $S\left(\alpha_{1}\right)$ not containing $p$. Using Lemma 3 , we obtain a $k$ torus $A_{i}^{\prime}$, which we put into general position with respect to $\mathrm{Bd} V^{\prime}$, so that

\section{$\mathrm{Bd} A_{i}^{\prime} \cap \mathrm{Bd} V^{\prime} \subset \mathrm{Bd} A_{i}^{*} \cap \mathrm{Bd} V^{\prime}-\left\{\alpha_{1}\right\} \cdot$}

We may eliminate all our $\alpha$-curves in this way, and obtain a $k$-torus $A_{i}$. Clearly $A_{i}$ will meet our requirements: for $i=1$,

$$
\begin{aligned}
& O\left(A_{2}^{*}, A_{1}\right)=O\left(A_{2}^{*}, A_{1}^{*}\right) \neq 0, \text { and } \\
& A_{2}^{*} \subset \operatorname{Int}\left(V^{\prime} \cap V\right) \subset V^{\prime} \cup V \subset \operatorname{Int} A_{1},
\end{aligned}
$$

while for $i=2, O\left(A_{2}, A_{1}^{*}\right)=O\left(A_{2}^{*}, A_{1}^{*}\right)$, and

$$
A_{2} \subset \operatorname{Int}\left(V^{\prime} \cap V\right) \subset V^{\prime} \cup V \subset \operatorname{Int} A_{1}^{*} .
$$

$V$ and $V^{\prime}$ are therefore $k$-similar, and $V^{\prime} \in \mathscr{V}$ : we conclude that no curve of $\mathrm{Bd} U \cap \mathrm{Bd} V^{*}$ can be null-homologous on $\mathrm{Bd} U$.

(b) Suppose some curve of $\mathrm{Bd} U \cap \mathrm{Bd} V^{*}$ bounds a disc on $\mathrm{Bd} V^{*}$. Then interchanging $U$ and $V^{*}$ in the arguments a(i) and a(ii), it follows that this intersection curve also bounds a disc on $\mathrm{Bd} U$, which we have shown in (a) to be impossible. Therefore no intersection curve bounds a disc on either surface.

Hence there is an even number of curves of $\mathrm{Bd} U \cap \mathrm{Bd} V^{*}$, bounding parallel annuli on $\mathrm{Bd} U$ lying alternately inside and outside $V^{*}$, with similar annuli on $\mathrm{Bd} V^{*}$. We may therefore choose two curves $\sigma_{1}$ and $\sigma_{2}$ which bound an annulus $A \subset \mathrm{Bd} U$ which contains no other intersection curves and which lies entirely inside $V^{*}$. We will use 
the theorems of H. Schubert ([7], §§16,17).

(i) Suppose $\sigma_{1}$ is not a meridian of $\mathrm{Bd} V^{*}$. By Satz 1, p. 207, $A$ separates $V^{*}$ into two solid tori $T_{1}$ and $T_{2} ; T_{1}$ has nonzero order in $V^{*}$ because it has $\sigma_{1}$ as a core, and $T_{2}$ has order 1 in $V^{*} \cdot \kappa\left(V^{*}\right)$ (and therefore $\kappa(V)$ ) is a companion of both $\kappa\left(T_{1}\right)$ and $\kappa\left(T_{2}\right)$. We put $\mathrm{Bd} T_{1}$ and $\mathrm{Bd} T_{2}$ into general position with respect to $\mathrm{Bd} U$, by taking $T_{i}^{*}=T_{i}-\{$ an open regular neighbourhood of $A\}$. Then

$\left(\mathrm{Bd} T_{1}^{*} \cap \mathrm{Bd} U\right) \cup\left(\mathrm{Bd} T_{2}^{*} \cap \mathrm{Bd} U\right)=\mathrm{Bd} V^{*} \cap \operatorname{Bd} U-\left\{\sigma_{1}, \sigma_{2}\right\}$.

Now one of the tori, $T_{r}^{*}$ say, contains $p$, and we may use Lemma 4 to show that $T_{r}^{*}$ is a $k$-torus; moreover, we may choose a very small regular neighbourhood of $A$ and so ensure that $T_{r}^{*}$ contains $B_{2}$ in its interior. (Certainly $T_{r}^{*} \subset \operatorname{Int} B_{1}$.) Also, we may modify the arguments of part (a) to show that $V$ and $T_{r}^{*}$ are $k$-similar, i.e. that $T_{r}^{*} \in \mathscr{V}$.

But since

$$
\mathrm{Bd} T_{r}^{*} \cap \mathrm{Bd} U \subset \mathrm{Bd} V^{*} \cap \mathrm{Bd} U-\left\{\sigma_{1}, \sigma_{2}\right\},
$$

the existence of this $T_{r}^{*} \in \mathscr{V}$ contradicts the minimality assumption involved in our choice of $V^{*}$. The curves of $\mathrm{Bd} U \cap \mathrm{Bd} V^{*}$ must be meridians of $\mathrm{Bd} V^{*}$, therefore,

(ii) $\sigma_{1}$ is a meridian of $\mathrm{Bd} V^{*}$. According to Satz 2, p. 211, $A$ separates $V^{*}$ into a solid torus $T(A)$ which shares a meridian disc with $V^{*}$, and which has order one in $V^{*}$ (so $\kappa\left(T(A)\right.$ ) has $\kappa\left(V^{*}\right)$ as a factor, and hence has $\kappa(V)$ as a companion); and another space which is a solid torus iff $T(A)$ and $V^{*}$ are equally knotted. If $p$ lies in $T(A)$, we may proceed as in part (i) above to eliminate the curves of $\mathrm{Bd} A$ by putting $\mathrm{Bd} T(A)$ into general position with respect to $\mathrm{Bd} U$ but then we would obtain a $k$-torus $T^{*} \in \mathscr{V}$ which would contradict our choice of $V^{*}$.

We suppose therefore that $p$ lies in $V^{*}-T(A)$. Let $A^{\prime}$ be the annulus on $\mathrm{Bd} V^{*}$, bounded by $\sigma_{1}$ and $\sigma_{2}$, such that $A$ and $A^{\prime}$ together bound $\mathrm{Cl}\left(V^{*}-T(A)\right)$. Since $A \cup A^{\prime}$ separates $p$ from $\mathrm{Bd} E_{0}$, and both annuli meet $k$ in at most one point each, $k \cap\left(A \cup A^{\prime}\right)$ cannot be empty and in fact

$$
1 \leqq N\left(k \cap\left(A \cup A^{\prime}\right)\right) \leqq 2 .
$$

There are then three cases to consider:

$N(k \cap A)=0, N\left(k \cap A^{\prime}\right)=1$. In this case, the torus $T(A)$ does not meet $k$ on its boundary at all, because $k \cap \mathrm{Bd} V^{*}=k \cap A^{\prime}$, and $\mathrm{Bd} T(A)=A \cup\left(\mathrm{Bd} V^{*}-A^{\prime}\right)$. But $T(A)$ and $V^{*}$ share a meridian disc. By Lemma 1, there is therefore a subarc of $k$ inside $T(A)$ which does not meet $\operatorname{Bd} T(A)$ at all-since $p$ is a point of $k$ lying outside 
$T(A), k$ must be disconnected. This is impossible, so this combination of intersection numbers cannot occur if $p$ is to lie in $V^{*}-T(A)$.

$N(k \cap A)=N\left(k \cap A^{\prime}\right)=1 . \quad k$ is an arc which is wild at one endpoint, so $\nu\left(k \cap\left(A \cup A^{\prime}\right)\right)= \pm 1$; so $k$ meets $A \cup A^{\prime}$ in an odd number of points. This is impossible in this case, for $N\left(k \cap\left(A \cup A^{\prime}\right)\right)=2$.

$N(k \cap A)=1, N\left(k \cap A^{\prime}\right)=0$. Let $R$ be any other annulus on $\operatorname{Bd} U$, bounded by intersection curves and whose interior lies in $\operatorname{Int} V^{*}$, and let the annulus $R^{\prime}$ be chosen on $\mathrm{Bd} V^{*}$ so that $R \cup R^{\prime}=\mathrm{Bd}\left(V^{*}-\right.$ $T(R)$ ), where $T(R)$ is the solid torus given by Satz 2, p. 211. $k$ does not meet $R$ at all, since $R$ lies in the interior of $\mathrm{Bd} U-A$ and $k \cap$ Bd $U=k \cap A$. $k$ can meet $R^{\prime}$ in at most one point: if $k \cap R^{\prime}=\varnothing$, then $p$ cannot lie in $V^{*}-T(R)$ and we may eliminate the curves of Bd $R$ by cutting $V^{*}$ along $R$. If $k$ meets $R^{\prime}$ in one point, then we have $N(k \cap R)=0$ and $N\left(k \cap R^{\prime}\right)=1$, which, we have shown above, implies that $p$ must lie in $T(R)$. So again we may eliminate the curves of $\mathrm{Bd} R$.

$\mathrm{Bd} U \cap \mathrm{Bd} V^{*}$ must therefore consist of only the two curves $\sigma_{1}$ and $\sigma_{2}$. We set $A_{1}^{*}=\mathrm{Cl}\left(\mathrm{Bd} V^{*}-A^{\prime}\right)$, and $A_{2}=\mathrm{Cl}(\operatorname{Bd} U-A)$. Since

$$
A_{2} \cap \mathrm{Bd} V^{*}=\mathrm{Bd} A_{2}=\mathrm{Bd} A=\left\{\sigma_{1}, \sigma_{2}\right\},
$$

$A_{1}^{*} \cup A_{2}$ is the boundary of a solid torus $V^{\prime}$ (Satz 3, p. 215) which is easily shown to be a $k$-torus and $k$-similar to $V . V^{\prime}$ lies in the interior of $B_{1}$, and contains $B_{2}$ in its interior; $V^{*}$ has order one in $V^{\prime}$. Therefore if $V^{\prime \prime}$ is a sufficiently small closed regular neighbourhood of $V^{\prime}, V^{\prime \prime} \in \mathscr{V}$ if we can prove that $\kappa(V)$ is a companion of $\kappa\left(V^{\prime \prime}\right)$-we then note that $\mathrm{Bd} V^{\prime \prime}$ does not meet $\mathrm{Bd} U$ at all, so the existence of $V^{\prime \prime} \in \mathscr{V}$ will contradict our choice of $V^{*}$.

Now $\sigma_{1}$ is not a meridian of $\mathrm{Bd} U$, since it bounds a disc in $V^{*}$ (the common meridian disc of $V^{*}$ and $T(A)$, for example) which lies in $\mathrm{Cl}\left(E_{0}-U\right) . \quad \sigma_{1}$ is not null-homologous on $\mathrm{Bd} U$, by (a). Then by Satz 1, p. 207, $A^{\prime}$ separates $U$ into two solid tori, one of which is $\mathrm{Cl}\left(V^{\prime}-V^{*}\right)$. By Satz 2, p. 211, therefore $V^{\prime}$ and $V^{*}$ are equally knotted, so $\kappa\left(V^{\prime \prime}\right)=\kappa\left(V^{*}\right)$. Thus $\kappa(V)$ is a companion of $\kappa\left(V^{\prime \prime}\right)$ and $V^{\prime \prime} \in \mathscr{V}$.

Therefore no intersection curve $\sigma \subset \mathrm{Bd} U \cap \mathrm{Bd} V^{*}$ can be a meridian of $\mathrm{Bd} V^{*} ; \sigma$ cannot be null-homologous on $\mathrm{Bd} V^{*}$, by (a), and cannot fail to be a meridian of $\mathrm{Bd} V^{*}$, by (b)(i). The existence of any intersection curves at all leads to a contradiction, and we conclude that $\operatorname{Bd} V^{*} \cap \operatorname{Bd} U=\varnothing$ for this choice of $V^{*} \in \mathscr{Y}$. This proves the lemma.

3. Containing sequences. Theorem 1 below will be of use later 
in finding invariants of oriented local type for arcs with $P_{1}=1$ and $P_{0} \geqq 5$, as well as in this paper.

THEOREM 1. Let $V$ be a k-torus for an arc $k$ in $R^{3}$ which is locally tame modulo one endpoint $p$ at which $P_{1}(k, p)=1$ and $P_{0}(k$, $p) \geqq 5$, and let

$$
V=V_{n} \prec V_{n-1} \prec \cdots \prec V_{0} \subset E_{0}
$$

and

$$
V=U_{m} \prec U_{m-1} \prec \cdots \prec U_{0} \subset E_{0}
$$

be two containing sequences for $V$ in $E_{0}$. Then $m=n$, and there exists a containing sequence

$$
V=U_{m}^{*} \prec U_{m-1}^{*} \prec \cdots \prec U_{0}^{*} \subset E_{0}
$$

with the properties

(i) $B d U_{i}^{*} \cap B d V_{j}=\varnothing$ for all $i, j=0,1, \cdots, n-1$ and

(ii) for all $i, \kappa\left(U_{i}\right)$ is a companion of $\kappa\left(U_{i}^{*}\right)$, and $U_{i}^{*}$ and $U_{i}$ are k-similar.

Proof. The proof involves more complicated cutting and pasting arguments than the proof of Lemma 5, for instead of juggling two $k$-tori, we are juggling two sequences of $k$-tori simultaneously.

It is sufficient to prove that the assumption $n<m$ leads to a contradiction, for then a symmetric argument will show that it is impossible for $m$ to be less than $n$.

Let $\mathscr{C l}$ be the class of all containing sequences

$$
V=T_{m} \prec T_{m-1} \prec \cdots \prec T_{0} \subset E_{0}
$$

of length $m$ in $E_{0}$, such that the surfaces Bd $T_{i}$ are in general position with respect to the surfaces $\operatorname{Bd} V_{j}$, and for each $i, U_{i}$ and $T_{i}$ are $k$-similar and $\kappa\left(T_{i}\right)$ has $\kappa\left(U_{i}\right)$ as a companion. If $\delta$ is the minimum of the distances from $\mathrm{Bd} U_{i}$ to $\mathrm{Bd} U_{i+1}$, we may ensure that the sequence

$$
V=U_{m} \prec U_{m-1} \prec \cdots \prec U_{0} \subset E_{0}
$$

is an element of $\mathscr{M}$, by an ambient isotopy of $E_{0}$ which leaves everything fixed outside an open regular neighbourhood of $\mathrm{Bd} U_{0} \cup \mathrm{Bd} U_{1} \cup$ $\cdots \cup \mathrm{Bd} U_{m-1}$, and moves no point further than $\delta / 4$.

$\mathscr{C}$ is not empty, therefore, and we may choose a sequence

$$
V=U_{m}^{*} \prec U_{m-1}^{*} \prec \cdots \prec U_{0}^{*} \subset E_{0}
$$

in $\mathscr{C}$ such that the set $C=\left\{\mathrm{Bd} U_{i}^{*} \cap \mathrm{Bd} V_{j}: i=0,1, \cdots, m-1\right.$ and $j=0,1, \cdots, n-1\}$ consists of a minimal number of intersection 
curves. Our aim is to show that $C$ is empty.

(a) Suppose some $\sigma \in C$ bounds a disc on $\operatorname{Bd} U_{i}^{*}$, for some $i$; then we may choose an innermost intersection curve $\tau$ which bounds a disc $D \subset \mathrm{Bd} U_{i}^{*}$ containing no other intersection curves. Using Lemma 1 as in part (a) of the proof of Lemma 5, we see that $\tau$ also bounds a disc $D(\tau)$ on $\mathrm{Bd} V_{j}$ if $\tau \subset \mathrm{Bd} U_{i}^{*} \cap \mathrm{Bd} V_{j}$. There exists an index $h$ and a curve $\rho \subset \mathrm{Bd} U_{h}^{*} \cap \mathrm{Bd} V_{j}$ such that $\rho$ bounds a subdisc $D(\rho) \subset$ $D(\tau) \subset \mathrm{Bd} V_{j}$, and such that $D(\rho)$ contains no other intersection curves in its interior. $\rho$ also bounds a disc on the boundary of $U_{h}^{*}$.

Thus, if there is any curve which bounds a disc on a surface $\mathrm{Bd} U_{i}^{*}$ or $\mathrm{Bd} V_{j}$, there exists an index $h$ and a curve $\rho \subset \mathrm{Bd} U_{h}^{*} \cap \mathrm{Bd} V_{j}$ which bounds a disc $D(\rho)$ on $\mathrm{Bd} V_{j}$, not containing any other intersection curves, and bounding a disc $D^{*}$ on $\mathrm{Bd} U_{h}^{*}$.

$D(\rho) \cup D^{*}$ is a 2-sphere bounding a 3-cell $S$. Applying Lemma 3 (and general positioning), we obtain a $k$-torus $U_{h}^{\prime}$ with $\kappa\left(U_{h}^{\prime}\right)=\kappa\left(U_{h}^{*}\right)$. $\kappa\left(U_{h}\right)$ is therefore a companion of $\kappa\left(U_{h}^{\prime}\right)$, and we may also show, as in Lemma 5, that $U_{h}^{\prime}$ and $U_{h}$ are $k$-similar. Moreover, for each $s$, $\mathrm{Bd} U_{h}^{\prime}$ meets $\mathrm{Bd} V_{s}$ in fewer curves than does $\mathrm{Bd} U_{h}^{*}$, for $\mathrm{Bd} U_{h}^{\prime} \cap \mathrm{Bd} V_{s}$ consists of those curves of $\mathrm{Bd} U_{h}^{*} \cap \mathrm{Bd} V_{s}$ which do not lie in $D^{*}$-in particular, $\rho$ has been eliminated. Thus the sequence

(*) $\quad V=U_{m}^{*} \prec U_{m-1}^{*} \prec \cdots \prec U_{h+1}^{*} \prec U_{h}^{\prime} \prec U_{h-1}^{*} \prec \cdots \prec U_{0}^{*} \subset E_{0}$

meets $\left\{\mathrm{Bd} V_{j}\right\}$ on its boundary surfaces in fewer curves than our original sequence $\left\{\mathrm{Bd} U_{i}^{*}\right\}$. This will contradict our choice of the sequence $\left\{U_{i}^{*}\right\}$ if we can show that the sequence $\left(^{*}\right)$ lies in the class $\mathscr{C}$; it is only necessary to show that $\left(^{*}\right)$ is a containing sequence for $V$.

Suppose $h=0$, and let $U$ be any $k$-torus with $U_{0}^{\prime} \subset U \subset E_{0}$. If $U_{0}^{\prime} \supset U_{0}^{*}$, then $U_{0}^{\prime}$ has nonzero order in $U$ because $\left\{U_{i}^{*}\right\}$ is a containing sequence in $E_{0}$ and $O\left(U_{0}^{*}, U_{0}^{\prime}\right)=1$. If $U_{0}^{\prime} \subset U_{0}^{*}$, there exists a $k$-torus $U^{\prime}$ which contains $U_{0}^{\prime}$, is $k$-similar to $U$, and whose boundary does not meet $\operatorname{Bd} U_{0}^{*}$, by Lemma 5. By Lemma 2, $O\left(U_{0}^{\prime}, U^{\prime}\right)$ and $O\left(U_{0}^{\prime}, U\right)$ are zero or nonzero together: if $U^{\prime} \subset U_{0}^{*}$, then

$$
1=O\left(U_{0}^{\prime}, U_{0}^{*}\right)=O\left(U_{0}^{\prime}, U^{\prime}\right) \cdot O\left(U^{\prime}, U_{0}^{*}\right)
$$

so $U_{0}^{\prime}$ has nonzero order in $U^{\prime}$; while if $U^{\prime} \supset U_{0}^{*}, U_{0}^{\prime}$ has nonzero order in $U^{\prime}$ because

$$
O\left(U_{0}^{\prime}, U^{\prime}\right)=O\left(U_{0}^{\prime}, U_{0}^{*}\right) \cdot O\left(U_{0}^{*}, U^{\prime}\right)=O\left(U_{0}^{*}, U^{\prime}\right)
$$

and $U_{0}^{*}$ is the first term in a containing sequence in $E_{0}$. In either case, $U_{0}^{\prime}$ has nonzero order in $U$, and the sequence $\left(^{*}\right)$ lies in $\mathscr{M}$.

Suppose $h>0$. We need only consider the case $U_{h}^{\prime}=U_{h}^{*}$-\{an open regular neighbourhood of $S\}$, the other case is analogous. 
Let $U$ be a $k$-torus with $U_{h}^{\prime} \subset U \subset U_{h-1}^{*}$. By Lemma 5 , there exists a $k$-torus $U^{\prime}, k$-similar to $U$ with $U_{h}^{\prime} \subset U^{\prime} \subset U_{h-1}^{*}$, and whose boundary does not meet $\mathrm{Bd} U_{h}^{*}$. Then, by Lemma 2, $O\left(U_{h}^{\prime}, U\right)$ and $O\left(U_{h}^{\prime}, U^{\prime}\right)$ are either zero or nonzero together, and $O\left(U, U_{h-1}^{*}\right)$ and $O\left(U^{\prime}, U_{h-1}^{*}\right)$ are both zero or both nonzero.

If $U^{\prime} \subset U_{h}^{\prime}, U_{h}^{\prime}$ has order 1 in $U^{\prime}$ because $O\left(U_{h}^{\prime}, U_{h}^{*}\right)=1$. If $U^{\prime} \supset$ $U_{h}^{*}$, then either $U_{h}^{*}$ has zero order in $U^{\prime}$ and $U^{\prime}$ has nonzero order in $U_{h-1}^{*}$ (because $\left\{U_{i}^{*}\right\}$ is a containing sequence), or $O\left(U_{h}^{*}, U^{\prime}\right) \neq 0$. Thus $U$ either contains $U_{h}^{\prime}$ with nonzero order, or is contained in $U_{h-1}^{*}$ with nonzero order, by Lemma 2. A similar argument shows that if $U$ is any $k$-torus with $U_{h+1}^{*} \subset U \subset U_{h}^{\prime}$ then either $O\left(U_{h+1}^{*}, U\right) \neq 0$ or $O(U$, $\left.U_{h}^{\prime}\right) \neq 0$.

It follows that $\left(^{*}\right)$ is a containing sequence for $V$ in $E_{0}$, and its existence in $\mathscr{C}$ contradicts the choice of our original sequence $\left\{U_{i}^{*}\right\}$, so we conclude that no $\rho \in C$ can bound a disc on any of the surfaces $\mathrm{Bd} U_{i}^{*}$ or $\operatorname{Bd} V_{j}$.

(b) For each pair $i$ and $j$, therefore, there is an even number of intersection curves bounding parallel annuli on $\mathrm{Bd} V_{j}$ and bounding parallel annuli on $\mathrm{Bd} U_{i}^{*}$. We may therefore choose a pair of curves $\sigma_{1}$ and $\sigma_{2}$ which bound an annulus $A \subset \mathrm{Bd} V_{j}$ which contains no other intersection curves, and such that Int $A \subset \operatorname{Int} U_{h}^{*}$ for some $h$. Once again we shall use Schubert's theorems.

(i) $\sigma_{1}$ is not a meridian of $\mathrm{Bd} U_{h}^{*}$. We apply Satz 1, p. 207: A separates $U_{h}^{*}$ into two solid tori $T_{1}$ and $T_{2}$. $T_{1}$ has nonzero order in $U_{h}^{*}$ because it has $\sigma_{1}$ as a core, and $T_{2}$ has order 1 in $U_{h}^{*} ; \kappa\left(U_{h}^{*}\right)$ (and therefore $\kappa\left(U_{h}\right)$ ) is a companion of both $\kappa\left(T_{1}\right)$ and $\kappa\left(T_{2}\right)$. We put $\mathrm{Bd} T_{1}$ and $\mathrm{Bd} T_{2}$ into general position with respect to the surfaces $\left\{\mathrm{Bd} V_{s}\right\}$ by setting $T_{r}^{*}=T_{r^{-}}$\{an open regular neighbourhood of $\left.A\right\}$. Then

$\left(\mathrm{Bd} T_{1}^{*} \cap \mathrm{Bd} V_{s}\right) \cup\left(\mathrm{Bd} T_{2}^{*} \cap \mathrm{Bd} V_{s}\right)=\operatorname{Bd} U_{h}^{*} \cap \operatorname{Bd} V_{s}$, except when $s=j$, when

$$
\left(\mathrm{Bd} T_{1}^{*} \cap \mathrm{Bd} V_{j}\right) \cup\left(\mathrm{Bd} T_{2}^{*} \cap \mathrm{Bd} V_{j}\right)=\mathrm{Bd} U_{h}^{*} \cap \mathrm{Bd} V_{j}-\left\{\sigma_{1}, \sigma_{2}\right\} .
$$

Now one of these tori, say $T_{h}^{*}$, contains $U_{h+1}^{*}$ in its interior. By Lemma $4, T_{h}^{*}$ is a $k$-torus and, as in Lemma $5, T_{k}^{*}$ is $k$-similar to $U_{h}$. Further, because $T_{h}^{*}$ has nonzero order in $U_{h}^{*}, U_{h+1}^{*} \prec T_{h}^{*} \prec U_{h-1}^{*}$ and we prove, as in (a) above, that the sequence

$$
V=U_{m}^{*} \prec \cdots \prec U_{h+1}^{*} \prec T_{h}^{*} \prec U_{h-1}^{*} \prec \cdots \prec U_{0}^{*} \subset E_{0}
$$

in an element of $\mathscr{M}$. The $k$-tori in this sequence meet the surfaces $\left\{\mathrm{Bd} V_{j}\right\}$ on their boundaries in fewer curves than do the $k$-tori in our original sequence $\left\{U_{i}^{*}\right\}$ (the curves $\left\{\sigma_{1}, \sigma_{2}\right\}=\operatorname{Bd} A$ have been eliminated), 
and this contradicts the minimality assumption involved in our choice of $\left\{U_{i}^{*}\right\}$.

(ii) Therefore, if the family $C$ of intersection curves is not empty, the curves of $\mathrm{Bd} U_{h}^{*} \cap \mathrm{Bd} V_{j}$ must be meridians of $\mathrm{Bd} U_{h}^{*}$. We will show that this is impossible.

We show first that $\mathrm{Bd} V_{j}$ cannot meet $\mathrm{Bd} U_{h+1}^{*}$ if it meets $\mathrm{Bd} U_{h}^{*}$. For, if $\mathrm{Bd} V_{j} \cap \mathrm{Bd} U_{h+1}^{*}$ is not empty, its curves cannot bound discs on $\mathrm{Bd} U_{h+1}^{*}$, by (a), and cannot fail to be meridians of $\mathrm{Bd} U_{h+1}^{*}$, by part (i) above. Since the curves of $\mathrm{Bd} V_{j} \cap\left(\mathrm{Bd} U_{h}^{*} \cup \mathrm{Bd} U_{h+1}^{*}\right)$ bound parallel annuli on $\mathrm{Bd} V_{j}$, we may choose two curves $\alpha_{h}$ and $\alpha_{h+1}$ of $\mathrm{Bd} V_{j} \cap \mathrm{Bd} U_{k}^{*}$ and $\mathrm{Bd} V_{j} \cap \mathrm{Bd} U_{h+1}^{*}$ respectively, so that $\alpha_{l}$ is a meridian of $\mathrm{Bd} U_{l}^{*}$, and so that $\alpha_{h}$ and $\alpha_{h+1}$ together bound an annulus on $\mathrm{Bd} V_{j}$ whose interior lies in Int $\left(U_{h}^{*}-U_{h+1}^{*}\right)$. But Lemma 1 of [2] then shows that $U_{h+1}^{*}$ must have order one in $U_{h}^{*}$, contradicting the assumption that $U_{h+1}^{*}$ has zero order in $U_{h}^{*}$. This contradiction shows that $\mathrm{Bd} V_{j}$ cannot meet $\mathrm{Bd} U_{h+1}^{*}$ if it meets $\mathrm{Bd} U_{h}^{*}$.

Nor can $\mathrm{Bd} V_{j}$ meet $\mathrm{Bd} U_{h-1}^{*}$ if it meets $\mathrm{Bd} U_{h}^{*}$. We may choose two intersection curves $\alpha_{h}$, which is a meridian of $\mathrm{Bd} U_{h}^{*}$, and $\alpha_{h-1}$ on $\mathrm{Bd} U_{h-1}^{*}$, which together bound an annulus on $\mathrm{Bd} V_{j}$ whose interior lies in Int $\left(U_{h-1}^{*}-U_{h}^{*}\right) . \quad \alpha_{h}$ and $\alpha_{h-1}$ are therefore homologous in $U_{h-1}^{*}$; but $\alpha_{h}$ bounds a disc in $U_{h-1}^{*}$, so $\alpha_{h-1}$ is null-homologous in $U_{h-1}^{*}$. $\alpha_{h-1}$ is therefore either null-homologous on $\mathrm{Bd} U_{h-1}^{*}$ or a meridian of $\operatorname{Bd} U_{h-1}^{*}$ ([7], p. 164). By (a), $\alpha_{h-1}$ cannot bound a disc on $\operatorname{Bd} U_{h-1}^{*}$, so $\alpha_{h-1}$ is a meridian of $\mathrm{Bd} U_{h-1}^{*}$; this implies, by Lemma 1 of [2], that $U_{h}^{*}$ has order one in $U_{h-1}^{*}$, which is impossible. Therefore no such curve $\alpha_{h-1}$ exists, and $\mathrm{Bd} V_{j}$ cannot meet $\mathrm{Bd} U_{h-1}^{*}$ if it meets $\mathrm{Bd} U_{h}^{*}$.

If $\mathrm{Bd} V_{j} \cap \mathrm{Bd} U_{h}^{*}$ is not empty, it follows that $\mathrm{Bd} V_{j} \subset \operatorname{Int}\left(U_{h-1}^{*}-\right.$ $\left.U_{h+1}^{*}\right)$. We may therefore use the same cutting and pasting techniques as were used in the last part of the proof of Lemma 5 , to obtain a $k$-torus $U_{h}^{\prime}$ which is $k$-similar to $U_{h}$, such that $\kappa\left(U_{h}^{*}\right)$ is a factor of $\kappa\left(U_{h}^{\prime}\right)$, and such that $\mathrm{Bd} U_{h}^{\prime} \cap \operatorname{Bd} V_{s}=\operatorname{Bd} U_{h}^{*} \cap \operatorname{Bd} V_{s}$ except when $s=j$, when

$$
\mathrm{Bd} U_{h}^{\prime} \cap \mathrm{Bd} V_{j}=\varnothing .
$$

We can also show (as in part (a)), that the sequence

$$
V=U_{m}^{*} \prec \cdots \prec U_{h+1}^{*} \prec U_{h}^{\prime} \prec U_{h-1}^{*} \prec \cdots \prec U_{0}^{*} \subset E_{0}
$$

is a containing sequence in $\mathscr{M}$; since the $k$-tori in this sequence meet the surfaces $\left\{\mathrm{Bd} V_{s}\right\}$ in fewer curves on their boundaries than do the surfaces $\left\{\mathrm{Bd} U_{i}^{*}\right\}$, the existence of this sequence in $\mathscr{C}$ contradicts the minimality assumption involved in our choice of the sequence $\left\{U_{i}^{*}\right\}$. This contradiction ensures that $\mathrm{Bd} V_{j}$ does not meet $\mathrm{Bd} U_{h}^{*}$ at all.

(c) It follows from the above that the family $C$ of intersection 
curves must be empty, that is, that $\mathrm{Bd} U_{i}^{*} \cap \mathrm{Bd} V_{j}=\varnothing$ for all $i=$ $0,1, \cdots, m-1$ and $j=0,1, \cdots, n-1$. We then have two containing sequences for $V$ in $E_{0}$, viz

$$
V=V_{n} \prec V_{n-1} \prec \cdots \prec V_{0} \subset E_{0}
$$

and

$$
V=U_{m}^{*} \prec U_{m-1}^{*} \prec \cdots \prec U_{0}^{*} \subset E_{0}
$$

such that for each $i$ and $j$, either $V_{j}$ lies in the interior of $U_{i}^{*}$, or contains $U_{i}^{*}$ in its interior.

$U_{m}^{*}$ and $V_{n}$ both equal $V$, so suppose $V_{n-1}$ lies in the interior of $U_{m-1}^{*}$. Then

$$
U_{m}^{*}=V_{n} \prec V_{n-1} \subset U_{m-1}^{*},
$$

so $V_{n-1}$ has nonzero order in $U_{m-1}^{*}$ because the $\left\{U_{i}^{*}\right\}$ form a containing sequence. On the other hand, if $U_{m-1}^{*}$ lies in the interior of $V_{n-1}$, it has non-zero order in $V_{n-1}$, because the $\left\{V_{j}\right\}$ form a containing sequence. Proceeding in this way, we can show that for each $r=0,1, \cdots, n$, either $V_{n-r}$ has nonzero order in $U_{m-r}^{*}$, or $U_{m-r}^{*}$ has nonzero order in $V_{n-r}$ (whichever is applicable).

But $m-n \geqq 1$ by hypothesis, so $O\left(V_{0}, U_{m-n}^{*}\right) \neq 0$ implies

$$
V_{0} \subset U_{m-n}^{*} \prec U_{0}^{*} \subset E_{0},
$$

which contradicts our choice of $V_{0}$ as the first $k$-torus in a containing sequence for $V$ in $E_{0}$. If $U_{m-n}^{*}$ has nonzero order in $V_{0}$, on the other hand, then $V_{0}$ must lie in the interior of $U_{m-n-1}^{*}$, and in fact have zero order in $U_{m-n-1}^{*}$-again contradicting our choice of $V_{0}$.

The assumption $n<m$ therefore leads to a contradiction, and $n=m$.

4. Invariance of $k$-sequences. Theorem 2, p. 24 of [4], asserts that every arc with $P_{1}=1$ and $P_{0} \geqq 5$ has a $k$-sequence in some 3 -cell $E$. Unfortunately, there are two errors in the proof of that theorem one the bald assertion that every such are has a constructing sequence in some 3-cell, and the other a misuse of corollary 3 of $[2]^{2}$. The validity of Theorem 2 is therefore in doubt, and we are led to the following problems, for arcs with $P_{1}=1$ and $P_{0} \geqq 5$.

Problem 1. Find sufficient conditions for an arc to have a constructing sequence in some 3-cell $E$. If possible, exhibit an arc which has no constructing sequences at all.

2 The author wishes to thank Professor Smythe for bringing the first of these to his attention. 
A set of sufficient conditions will be given in a later paper in this series, dealing with "special" arcs (cf. [4], Chapters III and IV).

Problem 2. Does the existence of a constructing sequence imply the existence of a $k$-sequence?

The author suspects that Theorem 2 on p. 24 of [4] is still true, that is that every arc has a constructing sequence and therefore a $k$-sequence, but has been unable to turn his hand to a suitable proof.

It is worth noting, however, that if an arc $k_{1}$ has a $k_{1}$-sequence $E_{0} \supset V_{0} \succ V_{1} \succ_{1} V_{2} \succ \cdots$, and $k_{2}$ has the same nonoriented local type at $p_{2}$ as $k_{1}$ has at $p_{1}$, then $k_{2}$ has a $k_{2}$-sequence. For let $h, U_{1}, U_{2}$ be as in the definition of non-oriented local type ( $\left(1\right.$, no. 3). Since $\cap V_{i}=$ $\left\{p_{1}\right\}$, there exists an index $N$ such that $V_{N} \subset \operatorname{Int} U_{1}$; let $E_{N}$ be any 3 -cell in the interior of $V_{N}$, which contains $V_{N+1}$ in its interior. Then

$$
h\left(E_{N}\right) \supset h\left(V_{N+1}\right) \succ h\left(V_{N+2}\right) \succ \cdots
$$

is a $k_{2}$-sequence.

We come now to the invariance of $k$-sequences.

sequences in $E_{0}$. Tnen $U_{i}$ and $V_{i}$ are equivalently knotted, for all $i$.

Proof. Given $U_{0}$, there exists an index $s(0)=s$ and a $k$-torus $V_{s}$ lying in the interior of $U_{0}$. By Theorem 1 , there exist $s-1 k$-tori $T_{1}, \cdots, T_{s-1}$ such that

$$
V_{s} \prec V_{s-1} \prec \cdots \prec V_{0} \subset E_{0}
$$

and

$$
V_{s} \prec T_{s-1} \prec \cdots \prec T_{1} \prec U_{0} \subset E_{0}
$$

are containing sequences for $V_{s}$ in $E_{0}$, and $s k$-tori $T_{1}^{*}, \cdots, T_{s-1}^{*}, U_{0}^{*}$ such that

(i) $V_{s} \prec T_{s-1}^{*} \prec \cdots \prec T_{1}^{*} \prec U_{0}^{*} \subset E_{0}$ is a containing sequence for $V_{s}$ in $E_{0}$,

(ii) $\mathrm{Bd} U_{0}^{*}$ does not meet $\mathrm{Bd} V_{j}$ for any $j=0,1, \cdots, s$, and

(iii) $U_{0}^{*}$ and $U_{0}$ are $k$-similar, and $\kappa\left(U_{0}^{*}\right)$ has $\kappa\left(U_{0}\right)$ as a companion.

From (ii), it follows that either $U_{0}^{*}$ lies in the interior of $V_{0}$, or that $V_{0} \subset$ Int $U_{0}^{*}$. In the latter case, $V_{0}$ cannot have zero order in $U_{0}^{*}$, so $\kappa\left(U_{0}^{*}\right)$ is a companion of $\kappa\left(V_{0}\right)$, hence $\kappa\left(U_{0}\right)$ is a companion of $\kappa\left(V_{0}\right)$. If $U_{0}^{*} \subset$ Int $V_{0}$, (i) shows that $U_{0}^{*}$ has nonzero order in $V_{0}$, so $U_{0}^{*}$ and $V_{0}$ must be concentric because $\left\{V_{j}\right\}$ is a $k$-sequence. So $\kappa\left(U_{0}^{*}\right)=\kappa\left(V_{0}\right)$, by Theorem 3 of [2], and again $\kappa\left(U_{0}\right)$ is a companion of $\kappa\left(V_{0}\right)$.

On the other hand, we may use similar arguments to prove that $\kappa\left(V_{0}\right)$ is a companion of $\kappa\left(U_{0}\right)$. 
It follows that $\kappa\left(U_{0}\right)=\kappa\left(V_{0}\right)$. We next observe that for some $s(1), V_{s(1)}$ lies in the interior of $U_{1}$ : by exactly the same method, we show that $\kappa\left(U_{1}\right)=\kappa\left(V_{1}\right)$ and, proceeding inductively, that $\kappa\left(U_{i}\right)=\kappa\left(V_{i}\right)$ for all $i$.

The proof of the following theorem is similar to the proof of the main theorem of [6].

THEOREM 2. Let $k_{1}$ and $k_{2}$ be arcs in $R^{3}$, locally tame modulo endpoints $p_{1}$ and $p_{2}$ respectively, at which $P_{1}\left(k_{i}, p_{i}\right)=1$ and $P_{0}\left(k_{i}, p_{i}\right) \geqq$ 5. If $k_{1}$ has a $k_{1}$-sequence $U_{0} \succ U_{1} \succ U_{2} \succ \cdots$ in some 3-cell $E_{0}(1)$, and $k_{2}$ a $k_{2}$-sequence $V_{0} \succ V_{1}>V_{2}>\cdots$ in some 3-cell $E_{0}(2)$, and $k_{2}$ has the same non-oriented local type at $p_{2}$ as $k_{1}$ has at $p_{1}$, then the sequences $\left\{\kappa\left(U_{0}\right), \kappa\left(U_{1}\right), \cdots\right\}$ and $\left\{\kappa\left(V_{0}\right), \kappa\left(V_{1}\right), \cdots\right\}$ are cofinal.

Unfortunately, the $k$-sequence is too unwieldy an invariant to be of much use in distinguishing nearly polyhedral arcs. A later paper will develop an invariant of oriented local type which is much simpler to apply than the $k$-sequence invariant, and we will show how these invariants may be used to distinguish wild arcs. Meantime, we merely assert that $k$-sequences will distinguish the arc of Figure 3 from the arc of Figure 1 (a).

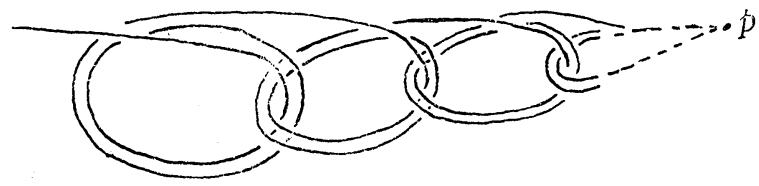

FIGURE 3

Added in proof. In connection with the note of $\S 1$, no. 2: Neville Smythe, Geoffrey Hemion and myself have shown that the

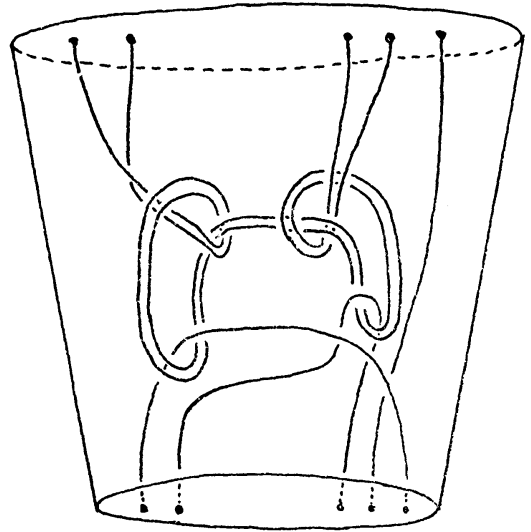

FIgURE 4 
arc constructed in the Fox-Artin manner from cylindrical sections of the type shown in Figure 4 , has $P_{1}=3$ but has penetration index 1 with respect to surfaces of genus 1 .

\section{REFERENCES}

1. B. J. Ball, Penetration Indices and Their Applications, in TOPOLOGY OF 3MANIFOLDS, ed. M. K. Fort, Jr., Prentice Hall, (1962), 37-39.

2. C. H. Edwards, Jr., Concentric solid tori in $S^{3}$, Trans. Amer. Math. Soc., 102 (1962), $1-17$.

3. D. W. Henderson, Self-unlinked simple closed curves, Trans. Amer. Math. Soc., 129 (1967), 322-343.

4. J. McPherson, Wild knots and arcs in a 3-manifold, Ph. D. thesis, University of New South Wales, 1970.

5. - Wild knots and arcs in a 3-manifold, in TOPOLOGY OF MANIFOLDS, ed. J. C. Cantrell and C. H. Edwards, Jr., Markham Publishing Company, 1970.

6. Wild arcs in three-space, 1: Families of Fox-Artin arcs, to appear in this Journal.

7. H. Schubert, Knoten und Vollringe, Acta Math. 90 (1953), 131-286.

8. H. Seifert und W. Threlfall, Lehrbuch der Topologie, Chelsea, N.Y., 1947.

Received September 14, 1971. The author would like to thank Professor Smythe for his comments and encouragement in the writing of the thesis, and especially for the use of a copy of his doctoral dissertation (Princeton University, 1965), from which was learnt the technique of "cutting and pasting" as applied to sequences of solid tori. The author would also like to thank Professor R. H. Fox for helpful comments on the thesis.

Most of the results in this paper were obtained while the author was at the University of Virginia. The author would like to thank Professor E. E. Floyd and the Mathematics Department of the University of Virginia, who provided a graduate assistantship and summer support for the 1968-69 academic year, and thus enabled me to continue my research under Professor Smythe after he had left the University of New South Wales.

The research for the thesis was supported in part by Australian Commonwealth Postgraduate Research Scholarship No. GN4118-7.

The Australian National University

Canberra, Australia 


\section{PACIFIC JOURNAL OF MATHEMATICS}

\section{EDITORS}

\section{H. SAMELSON}

Stanford University

Stanford, California 94305

C. R. Новву

University of Washington Seattle, Washington 98105

\section{J. DuGundJI}

Department of Mathematics University of Southern California Los Angeles, California 90007

RICHARD ARENS

University of California Los Angeles, California 90024

\section{ASSOCIATE EDITORS}
E. F. BECKENBACH
B. H. NeumanN
F. WOLF
K. YoSHIDA

\section{SUPPORTING INSTITUTIONS}

\author{
UNIVERSITY OF BRITISH COLUMBIA \\ CALIFORNIA INSTITUTE OF TECHNOLOGY \\ UNIVERSITY OF CALIFORNIA \\ MONTANA STATE UNIVERSITY \\ UNIVERSITY OF NEVADA \\ NEW MEXICO STATE UNIVERSITY \\ OREGON STATE UNIVERSITY \\ UNIVERSITY OF OREGON \\ OSAKA UNIVERSITY
}

\author{
UNIVERSITY OF SOUTHERN CALIFORNIA \\ STANFORD UNIVERSITY \\ UNIVERSITY OF TOKYO \\ UNIVERSITY OF UTAH \\ WASHINGTON STATE UNIVERSITY \\ UNIVERSITY OF WASHINGTON \\ $*{ }^{*}$
AMERICAN MATHEMATICAL SOCIETY
NAVAL WEAPONS CENTER
}

The Supporting Institutions listed above contribute to the cost of publication of this Journal, but they are not owners or publishers and have no responsibility for its content or policies.

Mathematical papers intended for publication in the Pacific Journal of Mathematics should be in typed form or offset-reproduced, (not dittoed), double spaced with large margins. Underline Greek letters in red, German in green, and script in blue. The first paragraph or two must be capable of being used separately as a synopsis of the entire paper. The editorial "we" must not be used in the synopsis, and items of the bibliography should not be cited there unless absolutely necessary, in which case they must be identified by author and Journal, rather than by item number. Manuscripts, in duplicate if possible, may be sent to any one of the four editors. Please classify according to the scheme of Math. Rev. Index to Vol, 39. All other communications to the editors should be addressed to the managing editor, Richard Arens, University of California, Los Angeles, California, 90024.

50 reprints are provided free for each article; additional copies may be obtained at cost in multiples of 50 .

The Pacific Journal of Mathematics is issued monthly as of January 1966. Regular subscription rate: $\$ 48.00$ a year (6 Vols., 12 issues). Special rate: $\$ 24.00$ a year to individual members of supporting institutions.

Subscriptions, orders for back numbers, and changes of address should be sent to Pacific Journal of Mathematics, 103 Highland Boulevard, Berkeley, California, 94708.

PUBLISHED BY PACIFIC JOURNAL OF MATHEMATICS, A NON-PROFIT CORPORATION

Printed at Kokusai Bunken Insatsusha (International Academic Printing Co., Ltd.), 270, 3-chome Totsuka-cho, Shinjuku-ku, Tokyo 160, Japan. 


\section{Pacific Journal of Mathematics}

\section{Vol. 44, No. $2 \quad$ June, 1973}

Tsuyoshi Andô, Closed range theorems for convex sets and linear liftings . . . . . . 393

Richard David Bourgin, Conically bounded sets in Banach spaces . . . . . . . . . 411

Robert Jay Buck, Hausdorff dimensions for compact sets in $R^{n} \ldots \ldots \ldots \ldots \ldots \ldots . \ldots 421$

Henry Cheng, A constructive Riemann mapping theorem ................ 435

David Fleming Dawson, Summability of subsequences and stretchings of

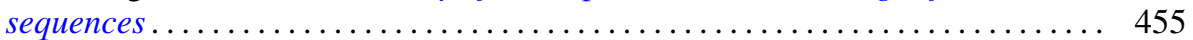

William Thomas Eaton, A two sided approximation theorem for 2-spheres ....... 461

Jay Paul Fillmore and John Herman Scheuneman, Fundamental groups of compact complete locally affine complex surfaces ....................... 487

Avner Friedman, Bounded entire solutions of elliptic equations . . . . . . . . . . . 497

Ronald Francis Gariepy, Multiplicity and the area of an $(n-1)$ continuous

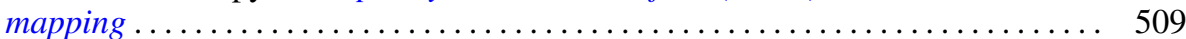

Andrew M. W. Glass, Archimedean extensions of directed interpolation groups . . . . 515

Morisuke Hasumi, Extreme points and unicity of extremum problems in $H^{1}$ on

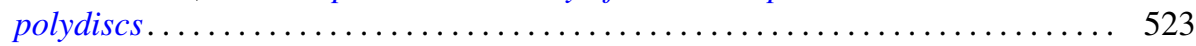

Trevor Ongley Hawkes, On the Fitting length of a soluble linear group . . . . . . 537

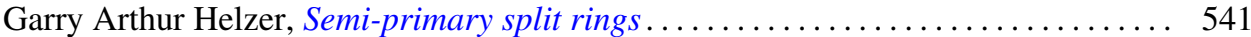

Melvin Hochster, Expanded radical ideals and semiregular ideals . . . . . . . . . 553

Keizō Kikuchi, Starlike and convex mappings in several complex variables . . . . . . 569

Charles Philip Lanski, On the relationship of a ring and the subring generated by its

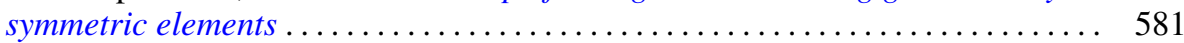

Jimmie Don Lawson, Intrinsic topologies in topological lattices and semilattices ........................................... 593

Roy Bruce Levow, Counterexamples to conjectures of Ryser and de Oliveira ...... 603

Arthur Larry Lieberman, Some representations of the automorphism group of an infinite continuous homogeneous measure algebra ..........

William George McArthur, $G_{\delta}$-diagonals and metrization theorems $\ldots .$.

James Murdoch McPherson, Wild arcs in three-space. II. An invariant of

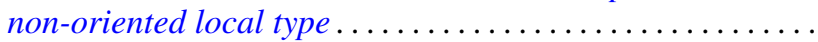

H. Millington and Maurice Sion, Inverse systems of group-valued measures ...

C. Edward Moore, Concrete semispaces and lexicographic separation of convex

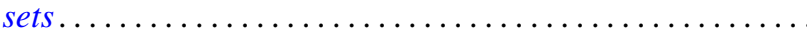

Jingyal Pak, Actions of torus $T^{n}$ on $(n+1)$-manifolds $M^{n+1}$.

Merrell Lee Patrick, Extensions of inequalities of the Laguerre and Turán type . . . . 675

Harold L. Peterson, Jr., Discontinuous characters and subgroups of finite index. . . . 683

S. P. Philipp, Abel summability of conjugate integrals . . . . . . . . . . . . . 693

R. B. Quintana and Charles R. B. Wright, On groups of exponent four satisfying an

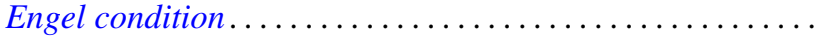

Marlon C. Rayburn, On Hausdorff compactifications. . . . . . . . . .

Martin G. Ribe, Necessary convexity conditions for the Hahn-Banach theorem in

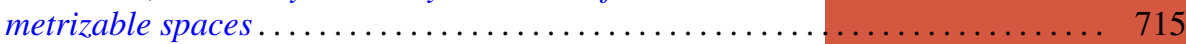

Ryōtarō Satō, On decomposition of transformations in infinite measure spaces .... 733

Peter Drummond Taylor, Subgradients of a convex function obtained from a

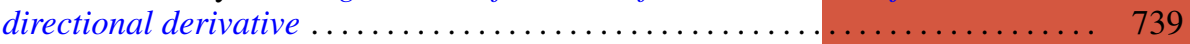

James William Thomas, A bifurcation theorem for $k$-set contractions . . . . . . . . 749 Clifford Edward Weil, A topological lemma and applications to real functions . . . . 757

Stephen Andrew Williams, A nonlinear elliptic boundary value problem . . ....... 767

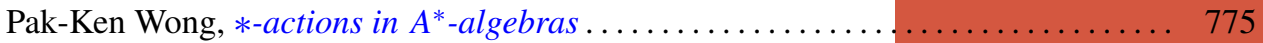

